\title{
Identifying microRNAs regulating B7-H3 in breast cancer: the clinical impact of microRNA-29c
}

M K Nygren ${ }^{1,9}$, C Tekle ${ }^{1,9}$, V A Ingebrigtsen ${ }^{1,2}$, R Mäkelä $^{3}$, M Krohn ${ }^{4,5}$, M R Aure ${ }^{4,5}$, C E Nunes-Xavier ${ }^{1}$, M Perälä ${ }^{3}$, T Tramm ${ }^{6}$, J Alsner ${ }^{6}$, J Overgaard ${ }^{6}$, J M Nesland ${ }^{2,7}$, E Borgen ${ }^{7}$, A-L Børresen-Dale ${ }^{4,5}, \varnothing$ Fodstad $^{1,2}$, K K Sahlberg ${ }^{4,5,8}$ and S-K Leivonen ${ }^{\star, 4,5,10}$

${ }^{1}$ Department of Tumor Biology, Institute of Cancer Research, Oslo University Hospital, N-0310 Oslo, Norway; ${ }^{2}$ Faculty of Medicine, Institute for Clinical Medicine, University of Oslo, N-0424 Oslo, Norway; ${ }^{3}$ Medical Biotechnology, VTT Technical Research Centre of Finland, Fl-20520 Turku, Finland; ${ }^{4}$ Department of Genetics, Institute of Cancer Research, Oslo University Hospital, N-0310 Oslo, Norway; ${ }^{5}$ The K.G. Jebsen Center for Breast Cancer Research, Institute for Clinical Medicine, Faculty of Medicine, University of Oslo, N-0424 Oslo, Norway; ${ }^{6}$ Department of Experimental Clinical Oncology, Aarhus University Hospital, DK-8000 Aarhus, Denmark; ${ }^{7}$ Department of Pathology, The Norwegian Radium Hospital, Oslo University Hospital, N-0424 Oslo, Norway and ${ }^{8}$ Department of Research, Vestre Viken Hospital Trust, N-3004 Drammen, Norway

Background: $\mathrm{B} 7-\mathrm{H} 3$, an immunoregulatory protein, is overexpressed in several cancers and is often associated with metastasis and poor prognosis. Here, our aim was to identify microRNAs (miRNAs) regulating B7-H3 and assess their potential prognostic implications in breast cancer.

Methods: MicroRNAs targeting B7-H3 were identified by transfecting two breast cancer cell lines with a library of 810 miRNA mimics and quantifying changes of B7-H3 protein levels using protein lysate microarrays. For validations we used western immunoblotting and $3^{\prime}$-UTR luciferase assays. Clinical significance of the miRNAs was assayed by analysing whether their expression levels correlated with outcome in two cohorts of breast cancer patients (142 and 81 patients).

Results: We identified nearly 50 miRNAs that downregulated B7-H3 protein levels. Western immunoblotting validated the impact of the 20 most effective miRNAs. Thirteen miRNAs (miR-214, miR-363*, miR-326, miR-940, miR-29c, miR-665, miR-34b*, miR-708, miR-601, miR-124a, miR-380-5p, miR-885-3p, and miR-593) targeted B7-H3 directly by binding to its 3'-UTR region. Finally, high expression of miR-29c was associated with a significant reduced risk of dying from breast cancer in both cohorts.

Conclusions: We identified miRNAs efficiently downregulating B7-H3 expression. The expression of miR-29c correlated with survival in breast cancer patients, suggesting a tumour suppressive role for this miRNA.

Breast cancer is the most common cancer in women worldwide, with about 1.38 million new cases and 458000 deaths each year (Ferlay et al, 2010). The majority of breast cancer morbidity and mortality is due to incurable metastatic disease that is highly resistant to conventional therapies. To reduce breast cancer mortality it is therefore essential to further elucidate the molecular mechanisms of breast cancer metastasis, and develop novel therapeutic approaches. Although decades of metastasis research

\footnotetext{
${ }^{*}$ Correspondence: Dr S-K Leivonen; E-mail: suvi-katri.leivonen@helsinki.fi

${ }^{9}$ These authors contributed equally to this work.

${ }^{10}$ Present address: Genome-Scale Biology Research Program, University of Helsinki, FI-00014 Helsinki, Finland.
}

Received 17 September 2013; revised 4 February 2014; accepted 7 February 2014; published online 27 February 2014 (C) 2014 Cancer Research UK. All rights reserved 0007 - 0920/14 
have provided considerable insight into the multistep metastatic process, there are still significant gaps in our knowledge.

Recently, it has been recognised that microRNAs (miRNAs) affect various steps in breast cancer metastasis including epithelialto-mesenchymal and mesenchymal-to-epithelial transition (EMT-MET transition), migration, invasion and angiogenesis and could represent potential therapeutic targets (Harquail et al, 2012; Wang et al, 2012). MicroRNAs are short non-coding RNAs that by targeting messenger RNAs (mRNAs) are key post-transcriptional regulators of gene expression (Ambros, 2001; Lai, 2002). By binding to the $3^{\prime}$-untranslated region ( $3^{\prime}$-UTR) of their target mRNA, miRNAs inhibit translation and/or promote mRNA degradation (Valencia-Sanchez et al, 2006). Consequently, they can act either as oncogenes or as tumour suppressors depending on their target mRNAs (Garzon et al, 2009; Shenouda and Alahari, 2009). The binding of an miRNA to an mRNA is not exclusive, as a single miRNA can target hundreds of mRNAs, and a single mRNA can be affected by multiple miRNAs (Selbach et al, 2008).

$\mathrm{B} 7-\mathrm{H} 3$, an immunoregulatory protein that belongs to the $\mathrm{B} 7$ family of T-cell co-regulatory molecules (Collins et al, 2005) is overexpressed in several different cancer forms and often associated with metastasis and poor prognosis (Sun et al, 2006; Roth et al, 2007; Crispen et al, 2008; Yamato et al, 2009; Zang et al, 2010; Katayama et al, 2011; Ingebrigtsen et al, 2012; Sun et al, 2012). In breast cancer, tumour B7-H3 expression is significantly correlated with more advanced disease and lymph node metastasis (Arigami et al, 2010; Liu et al, 2013). Whereas most reports emphasise the immunoregulatory function of $\mathrm{B} 7-\mathrm{H} 3$, we have demonstrated a non-immunological role of $\mathrm{B} 7-\mathrm{H} 3$ in cancer progression and metastasis, also supported by the work of Yuan et al (2011).

On the basis of the emerging evidence for miRNAs as regulators of cancer metastasis and our interest in B7-H3 as a metastasisassociated protein, we wanted to identify B7-H3-targeting miRNAs and to assess whether these have prognostic implications in breast cancer. It has previously been shown that miR-29 directly targets $\mathrm{B} 7-\mathrm{H} 3$ and leads to downregulation of the B7-H3 protein level in neuroblastoma, sarcoma and brain tumours (Xu et al, 2009) as well as in melanoma (Wang et al, 2013). In the present study, we evaluated the effects of 810 miRNAs on B7-H3 and phospho (p)-Stat3 protein levels, by utilising a protein lysate microarray (LMA) technology (Leivonen et al, 2009). We identified nearly 50 miRNAs downregulating $\mathrm{B} 7-\mathrm{H} 3$ and validated the 20 most effective miRNAs. We demonstrated that 13 of the 20 selected miRNAs, including miR-29c, targeted B7-H3 directly by binding to its $3^{\prime}$-UTR region. Finally, we showed that high expression of miR-29c is associated with increased survival in two cohorts of breast cancer patients.

\section{MATERIALS AND METHODS}

Cell culture and reagents. JIMT-1 cell line was procured from DSMZ (Brauenschweig, Germany) and grown in 1:1 DMEM/F12 (both from Mediatech, Inc., Manassas, VA, USA) media supplemented with $10 \%$ fetal bovine serum (FBS) (Atlanta Biologicals Inc., Lawrenceville, GA, USA), 1\% glutaMAX (Gibco, Paisly, UK), $10 \mu \mathrm{g} \mathrm{ml}^{-1}$ insulin (Sigma Chemicals, Perth, WA, Australia) and $1 \%$ penicillin-streptomycin (Lonza BioWhittaker, Walkersville, MD, USA). The KPL-4 cell line was a kind gift from Professor Junichi Kurebayashi (Kawasaki Medical School, Japan) and was grown in DMEM supplemented with 10\% FBS, $1 \%$ glutaMAX and $1 \%$ penicillin-streptomycin. Both cell lines were kept at $37{ }^{\circ} \mathrm{C}$ in $5 \% \mathrm{CO}_{2}$ and routinely tested for mycoplasma infections using the ATCC Mycoplasma detection kit (ATCC, Manassas, VA, USA).
Twenty different human mirVANA miRNA Mimics (hsa-miR-892a, hsa-miR-380-5p, hsa-miR-125b-2*, hsa-miR-363*, hsa-miR-940, hsa-miR-214, hsa-miR-34b*, hsa-miR-665, hsa-miR-593, hsa-miR29c, hsa-miR-555, hsa-miR-885-3p, hsa-miR-567, hsa-miR-297, hsa-miR-187-3p, hsa-miR-124a-1, hsa-miR-326, hsa-miR-601, hsa-miR-506 and hsa-miR-708) and the mirVANA miRNA mimic negative control \#1 were purchased from Life Technologies (Grand Island, NY, USA).

LMA screening and data analysis. The LMA screening has been described in detail previously (Leivonen et al, 2009, 2014). Briefly, JIMT- 1 and KPL- 4 were transfected with a human miRIDIAN microRNA mimic library v.10.1 (altogether 810 miRNA mimics) (Dharmacon, Lafayette, CO, USA) at a 20-nm concentration in 384 wells using siLentFect (Bio-Rad Laboratories, Hercules, CA, USA). After 48 or $72 \mathrm{~h}$ of incubation, cells were lysed and printed on nitrocellulose-coated microarray FAST slides (Whatman Inc., Florham Park, NJ, USA). B7-H3 and p-Stat3 protein expression were detected by staining the slides with a B7-H3 antibody (\#AF1027) and p-Stat3 antibody (\#9131) (both purchased from R\&D, Minneapolis, MN, USA), followed by exposure to Alexa Fluor 680-tagged secondary antibodies (Invitrogen Inc., Carlsbad, CA, USA). For total protein measurement, arrays were stained with Sypro Ruby Blot solution (Invitrogen). The slides were scanned with a Tecan LS400 (Tecan Inc., Durham, NC, USA) microarray scanner and an Odyssey Licor IR-scanner (LI-COR Biosciences, Lincoln, NE, USA) to detect Sypro, B7-H3 and p-Stat3 signals, respectively. Array-Pro Analyzer microarray analysis software (Median Cybernetics Inc., Bethesda, MD, USA) was used for analysing data. The $\mathrm{B} 7-\mathrm{H} 3$ and $\mathrm{p}-\mathrm{Stat} 3$ data were normalised to the Sypro total protein, $\log _{2}$ transformed and $z$-score standardized. $z$-scores $<-2.0$ were considered as a significant downregulation.

Transfection, SDS-PAGE and immunoblotting. To validate the LMA data, the cells were transfected with $20 \mathrm{~nm}$ mirVANA miRNA Mimics or $20 \mathrm{~nm}$ mirVANA miRNA mimic negative control \#1 using siLentFect according to the manufacturer's protocol. After $72 \mathrm{~h}$ the cells were lysed in $1 \times$ SDS sample buffer (NuPage, Life Technologies, Carlsbad, CA, USA) supplemented with reducing agent (NuPage, Life Technologies). The samples were heated at $95^{\circ} \mathrm{C}$ for $10 \mathrm{~min}$ and then separated by SDS-polyacrylamide gel electrophoresis (SDS-PAGE) in 4-12\% gels (Life Technologies) and transferred onto PVDF membranes (Bio-Rad Laboratories). Blotted membranes were blocked in Tris-buffered saline with $0.1 \%$ Tween-20 (TBST) and 5\% non-fat dry milk for $1 \mathrm{~h}$ at room temperature and probed overnight at $4{ }^{\circ} \mathrm{C}$ with specific monoclonal or polyclonal antibodies (B7-H3, phospho-Stat3 or total Stat3) in TBST with 5\% non-fat dry milk or bovine serum albumin. Anti- $\beta$-actin (\#sc-1616, Santa Cruz Biotechnology, Santa Cruz, CA, USA) was used as a loading control. The horseradish peroxidase conjugated secondary antibodies were all from Dako (Glostrup, Denmark). Signals were developed with Super Signal West Dura Extended Duration Substrate (Pierce, Thermo Scientific, Rockford, IL, USA) and measured by the digital image station (Kodak, Rochester, NY, USA).

$3^{\prime}$-UTR reporter constructs and luciferase assays. The $\mathrm{B} 7-\mathrm{H} 3$ 3'-UTR GoClone contruct was purchased from SwitchGear Genomics (MenloPark, CA, USA). An empty 3'-UTR GoClone (SwitchGear) was used as a positive control. For luciferase assays, JIMT-1 cells (10000 per well) were plated onto 96-well plates $24 \mathrm{~h}$ before transfection. The cells were co-transfected with $100 \mathrm{ng}$ of B7-H3 $3^{\prime}$-UTR GoClone construct and $50 \mathrm{~nm}$ mirVANA miRNA Mimic using Lipofectamine 2000 (Life Technologies) as a transfection reagent according to the manufacturer's protocol. Luciferase activity was assayed $24 \mathrm{~h}$ after transfection with a Dual-Glo Luciferase Assay System (Promega Corp., Madison, WI, USA) and 
was measured with a Wallac Victor2 plate reader (Perkin Elmer, Waltham, MA, USA).

Patient material. The DBCGbc patient material included 142 tumours from high-risk Danish breast cancer patients from the DBCG82bc cohort, with $>20$ years of follow-up information. Premenopausal women (DBCGb protocol) were randomised to receive radiation therapy and $\mathrm{CMF}$ (cyclophosphamide, methotrexate, fluorouracil; eight cycles) or only CMF chemotherapy (nine cycles). Postmenopausal women (DBCGc protocol) were randomised to receive radiation therapy + tamoxifen $(30 \mathrm{mg}$ daily for 1 year) or tamoxifen alone (Kyndi et al, 2008). Oral informed consent was mandatory, and the study of the DBCG82bc cohort was approved by the Regional Ethical Committee (Journal number 20030263). Gene expression, miRNA expression, ER status, HER2 status and TP53 mutation status were used for analysis on this data set (Kyndi et al, 2008; Myhre et al, 2010; Aure et al, 2013).

The MicMa patient material consisted of 101 stage I and II breast cancer patients diagnosed in 1995-1998 with a median time of follow-up of 85 months. These were part of a larger patient material consisting of 920 patients with clinical information of disseminated tumour cell status (Wiedswang et al, 2003). The study was approved by the regional ethical committee (S-97103), and all patients have given informed written consent. Gene expression, miRNA expression, ER status, HER2 status and TP53 mutation status have been described before (Enerly et al, 2011). For 81 samples, full clinical information was available and those were used for the survival analyses.

MicroRNA expression analyses. For MicMa, the tumour RNA from fresh frozen samples was isolated using TRIzol reagent (Invitrogen) (Enerly et al, 2011), and for DBCGbc with the Qiagen Midi kit Extraction column procedure (Qiagen $\mathrm{GmbH}$, Hilden, Germany) (Aure et al, 2013). The miRNA expression profiling for both cohorts was performed with the $8 \times 15 \mathrm{k}$ 'Human miRNA Microarray Kit (V2)' (design id 019118) from Agilent (Agilent Technologies, Santa Clara, CA, USA). Briefly, $100 \mathrm{ng}$ total RNA was dephosphorylated, labelled and hybridised for $20 \mathrm{~h}$ following the manufacturer's protocol. Scanning was performed on Agilent Scanner G2565A, signals were extracted using Feature Extraction v.9.5 and the subsequent data processing was performed using the GeneSpring software v.12.0 (Agilent Technologies). For normalisation, the expression values were $\log _{2}$ transformed and normalised to the 90th percentile. MicroRNAs that were detected in $<10 \%$ of the samples were excluded. The miRNA data have been previously published in the Gene Expression Omnibus (GEO) with accession number GSE19536 for the MicMa data (Enerly et al, 2011), and GSE46934 for the DBCGbc data (Aure et al, 2013).

Quantitative RT-PCR analyses. Total RNAs from JIMT-1 and KPL-4 cells were isolated with the MiRVANA kit (Ambion, Austin, TX, USA) according to the manufacturer's instructions. Absolute quantification of B7-H3 levels was performed using the TaqMan Gene Expression assay Hs00987207_m1 (Applied Biosystems, Foster City, CA, USA). Beta-actin was used as an endogenous control (assay 4352935E). Absolute quantification of miR-29c was performed using the TaqMan MicroRNA assay 000587 (Applied Biosystems) with RNU6B as an endogenous control (assay 001093). The standard curves were set up as a two-fold dilution series in triplicates of known concentrations of FirstChoice Human Breast Total RNA (Applied Biosystems). RT-PCR reactions were carried out using the manufacturer's recommendation. In brief, 5 ng of total RNA was reverse transcribed using the High Capacity cDNA Reverse Transcription Kit with RNase inhibitor (Applied Biosystems) for B7-H3 and $\beta$-actin, and the TaqMan MicroRNA Reverse Transcription kit (Applied Biosystems) with miRNAspecific RT-primers (Applied Biosystems) for miR-29c and RNU6B. Quantitative real-time PCR was performed following the manufacturer's recommendation in triplicates on a 7900 HT Fast Real-Time PCR System (Applied Biosystems) with a standard absolute quantification thermal cycling program. Cycle threshold $\left(C_{t}\right)$ values and absolute quantification were obtained using the SDS 2.3 software (Applied Biosystems).

Statistical analysis. The in vitro experiments were performed a minimum of four times, and all data are shown as mean \pm s.e.m. The data were analysed for statistical significance using an unpaired Student's $t$-test. Univariate survival analysis was performed according to the Kaplan-Meier method, and survival was compared using the log-rank test. Statistical significance of miR-29c expression between molecular breast cancer subtypes and histologic grade was determined by the Kruskal-Wallis test. Correlation between miR-29c expression and B7-H3 mRNA expression was calculated using the Spearman Rank method, and association of miR-29c expression with the B7-H3 status was determined by the Mann-Whitney test. The SPSS version 18 software (SPSS Inc., Chicago, IL, USA) and STATA (STATA Corp., College Station, TX, USA) were used for statistical calculations. For all the experiments, any $P$-value below 0.05 was considered statistically significant.

\section{RESULTS}

Protein LMA to identify miRNAs regulating $\mathrm{B} 7-\mathrm{H} 3$ in breast cancer cell lines. We applied protein LMA screening (Leivonen et al, 2009) to identify B7-H3-regulating miRNAs in two breast cancer cell lines, KPL-4 and JIMT-1, which express B7-H3 at relatively high levels. The cells were transfected with a miRNA mimic library containing 810 miRNAs. After 48 and $72 \mathrm{~h}$ of incubation, the cells were lysed, printed on nitrocellulose-coated slides and stained with a specific antibody against B7-H3. As we previously have demonstrated that silencing B7-H3 affects the level of p-Stat3 (Liu et al, 2011), we also stained the slides with a specific p-Stat3 antibody. In addition, the slides were counterstained with Sypro Ruby to normalise the levels of $\mathrm{B} 7-\mathrm{H} 3 / \mathrm{p}-\mathrm{Stat} 3$ to total protein. The whole screening data with normalised $z$-scores are provided as a Supplementary Table S1.

In the JIMT-1 cell line, we initially identified 46 miRNAs that significantly reduced the protein expression of B7-H3 at a 48-hour time point $(z$-score $<-2.0)$, compared with 48 at $72 \mathrm{~h}$ (Supplementary Table S1). For the KPL-4 cell line, 49 miRNAs significantly reduced the expression of B7-H3 after $48 \mathrm{~h}$ and 49 after $72 \mathrm{~h}$ (Supplementary Table S1). Interestingly, there were also miRNAs efficiently upregulating $\mathrm{B} 7-\mathrm{H} 3$ in both cell lines, for instance miR-518a-5p. However, as our aim was to find negative regulators of $\mathrm{B} 7-\mathrm{H} 3$, we did not select the upregulating miRNAs for further analyses.

We also identified 37 miRNAs that reduced p-Stat 3 protein level after $48 \mathrm{~h}$ and 27 after $72 \mathrm{~h}$ (KPL-4, Supplementary Table S1). MiR-29c, which previously has been shown to regulate B7-H3 in neuroblastoma, sarcoma and brain cancers (Xu et al, 2009) was among the strongest hits in our screen.

Furthermore, we showed that there was a correlation between the $z$-score values after both 48 (Figure 1A) and $72 \mathrm{~h}$ (Figure 1B) for the JIMT-1 and the KPL- 4 cell lines. Thus, 33 of 46 and 37 of 48 (48 and $72 \mathrm{~h}$, respectively) (Supplementary Table S1, marked in bold) of the miRNAs effective in JIMT-1 cells were effective in KPL-4 cells as well. Conversely, 33 of 49 and 37 of 49 (48 and 72 h, respectively) (Supplementary Table S1, marked in bold) of the miRNAs with significant effects in KPL-4 cells were effective in JIMT-1 cells. Notably, a strong relationship between $z$-score values for B7-H3 and p-Stat 3 expression after both 48 and $72 \mathrm{~h}$ was found (Supplementary Figure S1A and S1B). 


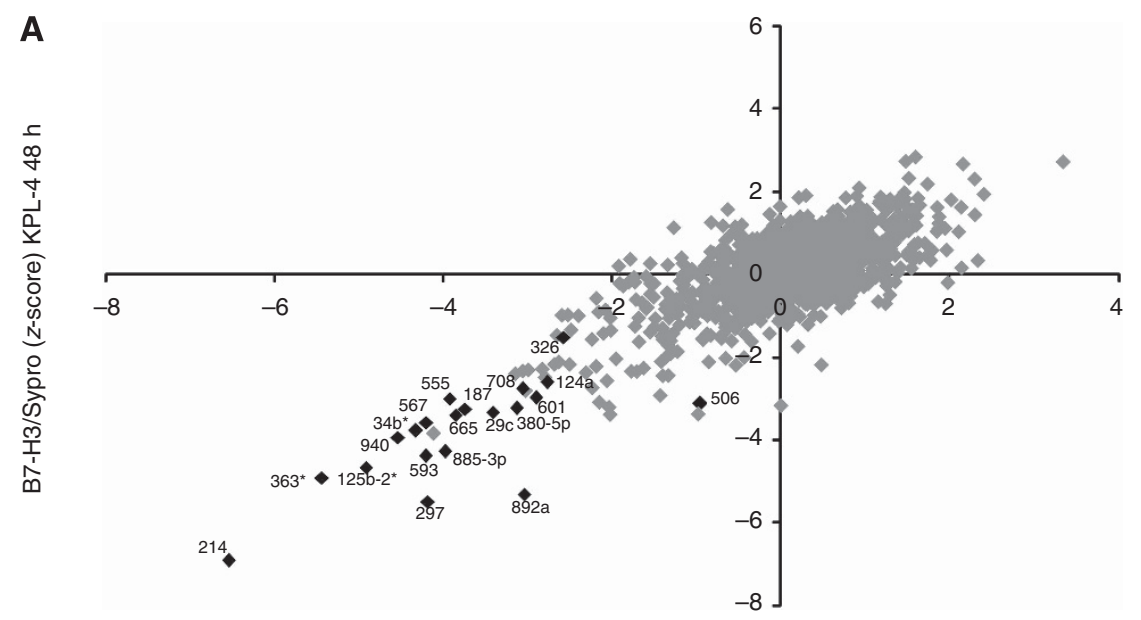

B7-H3/Sypro (z-score) JIMT-1 48 h

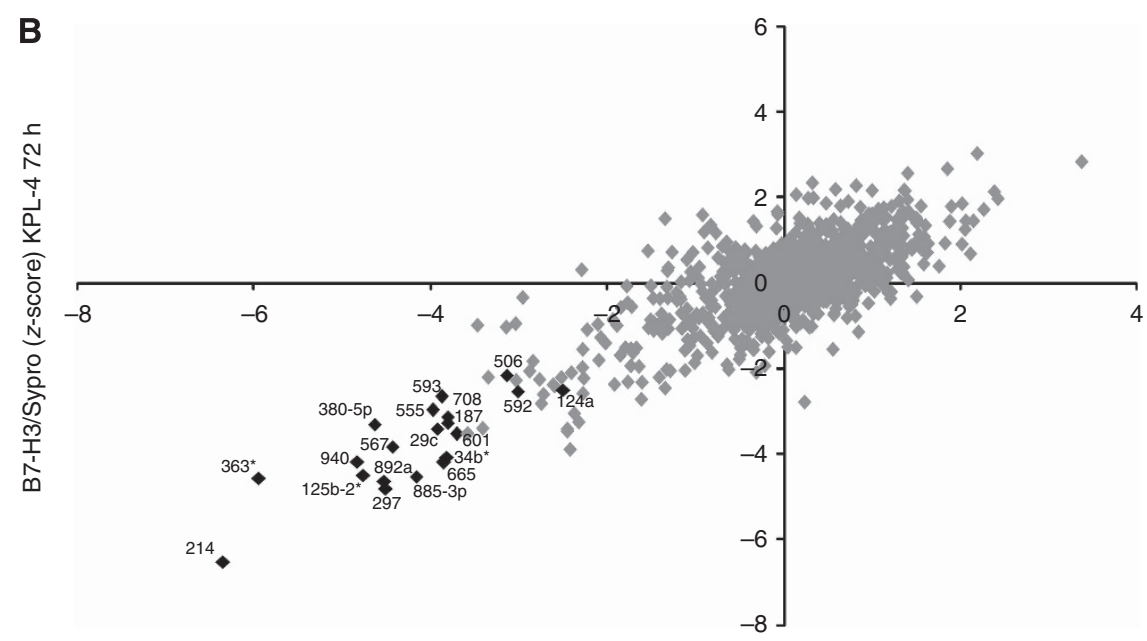

B7-H3/Sypro (z-score) JIMT-1 72 h

Figure 1. Protein lysate microarray (LMA) screen for identifying microRNAs (miRNAs) regulating B7-H3. (A) A scatter plot of a 48-h screen (JIMT-1 vs KPL-4). (B) A scatter plot of a 72-h screen (JIMT-1 vs KPL-4). Black spots indicate miRNAs that were selected for further validation by western immunoblotting and are marked with numbers representing miRNA names.

Validation of screening data by assessment of the $\mathrm{B} 7-\mathrm{H} 3$ and phosphorylated Stat 3 protein levels. To validate the primary screening data, we selected the 17 most effective miRNAs downregulating B7-H3 (Table 1). In addition, we decided to validate miR-326, miR-505 and miR-124a based on their strong negative effect on the p-Stat 3 levels in the KPL-4 cell line. Notably, we were not able to detect p-Stat 3 in the JIMT-1 cell line in the screen.

The 20 selected miRNAs were transfected into the JIMT-1 and the KPL- 4 cells, and after $72 \mathrm{~h}$ the cells were lysed and the protein levels of B7-H3 and p-Stat3 were analysed by western immunoblotting. In the JIMT-1 cells, we demonstrated that all the miRNAs downregulated $\mathrm{B} 7-\mathrm{H} 3$ protein expression as compared with negative control scrambled miRNAs (Figure 2A; Supplementary Figure S2). Consistent with the LMA results, p-Stat3 levels in JIMT-1 cells were low (results not shown). For the KPL-4 cell line, most of the miRNAs caused a reduction in the B7-H3 protein level (Figure 2B; Supplementary Figure S2). The level of downregulation varied among the different miRNAs, the miR-506 and miR-187-3p having the weakest influence on the B7-H3 level.

Finally, we investigated the phosphorylation status of Stat3 in the KPL- 4 cell line and observed that 11 of the 20 miRNAs also reduced the level of p-Stat3. MiR-297, miR-34b*, miR-124a and
miR-380-5p had a very weak or no influence on the $\mathrm{p}-\mathrm{Stat} 3$ protein level although the B7-H3 protein level was reduced. This was in concordance with the LMA data and it might reflect that these miRNAs may exert their effects on $\mathrm{B} 7-\mathrm{H} 3$ via other target genes than those affecting Stat3.

Validating miRNAs binding directly to the B7-H3 $3^{\prime}$-UTR. All the 20 miRNAs that were selected for validation were investigated by miRanda (Betel et al, 2008) and TargetScan algorithms (Grimson et al, 2007) for their potential interaction with B7-H3 $3^{\prime}$-UTR. We found that 13 of the selected miRNAs were predicted to bind to B7-H3 3'-UTR (Table 2), which contains a sequence of $\sim 1500$ bases.

To examine whether the 20 miRNAs actually could bind to the B7-H3 3'-UTR, we took advantage of the LightSwitch miRNA Target Validation System (SwitchGear). We used a luciferase reporter construct (LightSwitch $3^{\prime}$-UTR reporter GoClone) with B7-H3 3'-UTR sequence using the novel RenSP luciferase technology (SwitchGear). The reporter was co-transfected into JIMT-1 cells with miRNAs and the luciferase activity was measured after $24 \mathrm{~h}$. Transfection of miR-214, miR-363*, mir-326, miR-940, miR-29c, miR-665, miR-34b*, miR-708, miR-601, miR-124a, miR-380-5p, miR-885-3p and miR-593 significantly inhibited the 


\begin{tabular}{|c|c|c|c|c|c|c|}
\hline \multirow{2}{*}{\multicolumn{3}{|c|}{$\begin{array}{l}\text { Table 1. B7-H3 downregulation ( } z \text { - } \\
\text { with p-Stat3 downregulation ( } z \text {-sco } \\
\text { validation } \\
\qquad \begin{array}{c}\text { B7-H3/sypro } \\
\mathbf{z} \text {-score }\end{array} \\
\text { JIMT-1 }\end{array}$}} & \multirow{2}{*}{\multicolumn{2}{|c|}{\begin{tabular}{|c|}
$\begin{array}{c}\text { B7-H3/sypro } \\
\text { z-score }\end{array}$ \\
KPL-4 \\
\end{tabular}}} & \multicolumn{2}{|c|}{$\begin{array}{c}\text { p-Stat3/sypro } \\
\text { z-score }\end{array}$} \\
\hline & & & & & $\mathrm{KP}$ & \\
\hline miRNA & $48 \mathrm{~h}$ & $72 \mathrm{~h}$ & $48 \mathrm{~h}$ & $72 \mathrm{~h}$ & $48 \mathrm{~h}$ & $72 \mathrm{~h}$ \\
\hline hsa-miR-214 & -6.54 & -6.35 & -6.92 & -6.53 & -2.16 & -2.28 \\
\hline hsa-miR-363* & -5.43 & -5.94 & -4.90 & -4.57 & -1.28 & -2.58 \\
\hline hsa-miR-326 & -2.64 & -3.15 & -1.49 & -1.04 & -3.58 & -3.85 \\
\hline hsa-miR-940 & -4.53 & -4.83 & -3.95 & -4.19 & 1.16 & 0.78 \\
\hline hsa-miR-125b-2* & -4.92 & -4.76 & -4.65 & -4.50 & -1.24 & -0.04 \\
\hline hsa-miR-380-5p & -3.13 & -4.64 & -3.24 & -3.31 & -0.25 & 0.46 \\
\hline hsa-miR-892a & -3.04 & -4.52 & -5.31 & -4.63 & -1.21 & -2.46 \\
\hline hsa-miR-297 & -4.18 & -4.51 & -5.48 & -4.80 & 0.39 & -0.07 \\
\hline hsa-miR-567 & -4.20 & -4.43 & -3.59 & -3.83 & -1.93 & -0.85 \\
\hline hsa-miR-885-3p & -3.98 & -4.17 & -4.28 & -4.53 & -0.16 & 0.21 \\
\hline hsa-miR-555 & -3.92 & -3.97 & -3.00 & -2.98 & -0.32 & -0.28 \\
\hline hsa-miR-29c & -3.41 & -3.92 & -3.32 & -3.41 & -1.19 & -2.79 \\
\hline hsa-miR-593 & -4.21 & -3.87 & -4.39 & -2.66 & 0.37 & 0.29 \\
\hline hsa-miR-665 & -3.86 & -3.85 & -3.41 & -4.20 & 0.91 & -1.14 \\
\hline hsa-miR-34b* & -4.32 & -3.82 & -3.78 & -4.07 & 1.64 & 1.59 \\
\hline hsa-miR-187-3p & -3.74 & -3.81 & -3.26 & -3.13 & -0.14 & -0.91 \\
\hline hsa-miR-708 & -3.05 & -3.80 & -2.77 & -3.28 & -2.94 & -2.64 \\
\hline hsa-miR-506 & -0.95 & -3.14 & -3.12 & -2.17 & -1.34 & -2.47 \\
\hline hsa-miR-601 & -2.90 & -3.70 & -2.98 & -3.53 & 0.02 & -1.08 \\
\hline hsa-miR-124a & -2.78 & -2.50 & -2.50 & -2.51 & -0.37 & -1.46 \\
\hline
\end{tabular}

luciferase activity of the construct containing the B7-H3 3'-UTR compared with scrambled miRNA-transfected controls (Figure 3), indicating that these miRNAs directly bind to the B7-H3 $3^{\prime}$-UTR.

High miR-29c expression is associated with increased breast cancer-related survival. To determine the possible prognostic role of B7-H3-regulating miRNAs, we analysed whether their expression level correlates with breast cancer prognosis in two independent patient cohorts. Of the selected 20 miRNAs, 10 (miR-214, -940, -125b-2*, -885, -29c, -665, -34b* $,-708,-601$, and -326) were expressed in the tumour samples in both clinical cohorts and were included in the analyses. Distribution of the clinicopathological parameters of the patients in the two cohorts is given in Supplementary Table S2. Using breast cancer death as an end point, we first checked whether any of the 10 miRNAs were of significant prognostic importance. This was screened by separating the individual miRNA values in quartiles. The analysis was first performed in a cohort of 142 patients from DBCG82bc study (Kyndi et al, 2008; Myhre et al, 2010; Aure et al, 2013). One miRNA (miR-29c) was found to have prognostic value that was associated with a high miR-29c expression (Figure 4A). This was further explored by a spline analysis aimed to optimise the best cut point between high and low values. This was found to be a value of 3.35 (by coincidence almost identical with the cut point between the third and fourth quartile). On the basis of this cut point, we then performed a univariate analysis and found a hazard ratio (HR): 0.37 (0.20-0.69, 95\% confidence level), with $P=0.001$ for breast cancer death. After adjusting for age, tumour size, nodal status, HER2 and ER status, the HR was: 0.46 (0.25-0.86), $P=0.008$. In addition, when we analysed miR-29c expression in relation to distant metastasis, we found that higher miR-29c expression was associated with lower risk of developing distant metastasis (HR: $0.48(0.29-0.80)$ ).

Next, we validated this finding in 81 stage I and II breast cancer patients (MicMa) (Enerly et al, 2011). Using the same approach as above, we independently confirmed the superior prognostic value of high miR-29c expression (Figure 4B) with a univariate HR of 0.39 (0.15-0.98), $P=0.04$, and a multivariate adjusted HR of 0.36 (0.14-0.93), $P=0.02$, for breast cancer death.

As we had demonstrated that miR-29c binds to B7-H3 $33^{\prime}-\mathrm{UTR}$ and downregulates the $\mathrm{B} 7-\mathrm{H} 3$ protein in breast cancer cell lines, we investigated whether the observed association between high miR-29c and improved prognosis was related to the reduced level of B7-H3 in the tumours. When we analysed a potential correlation between B7-H3 transcript and miRNA expression levels using the Spearman correlation in the MicMa cohort, we found a significant negative correlation $(r=-0.268 ; P=0.008)$ between B7-H3 mRNA and miR-29c (Supplementary Figure S3). In the cell lines, endogenous miR-29c levels were low, whereas B7-H3 had relatively high expression (Supplementary Figure S4).

HER2 expression and loss of oestrogen receptor (ER) expression both indicate more aggressive breast cancer, whereas inactivation of the p53 tumour suppressor is common in several cancer forms including breast cancer. We therefore investigated whether any of these parameters were associated with miR-29c expression, by comparing the miR-29c mean value in TP53 normal and mutated tumours, in HER2-positive and HER2-negative tumours and in ER-positive and ER-negative tumours. In both cohorts, we found that miR-29c had lower expression in tumours with TP53 mutation (MicMa $P<0.001$, DBCGbc $P=0.048$ ), HER2 overexpression (MicMa $P=0.037$, DBCGbc $P=0.004$ ) and loss of ER expression $(P<0.001$ both cohorts) (Supplementary Table S3). In addition, miR-29c had lower expression in higher grade tumours and in the basal subtype (Supplementary Figure S5), which fits well with the fact that higher miR-29c expression predicts better survival.

\section{DISCUSSION}

In this study, we present the results of a high-throughput miRNA gain-of-function screening, followed by protein LMA to identify and validate miRNAs that affect the expression of B7-H3 in breast cancer cells. Except for miR-29c, which has been reported to directly target $\mathrm{B} 7-\mathrm{H} 3$ in neuroblastomas, sarcomas and brain tumours (Xu et al, 2009) and in melanoma (Wang et al, 2013), and miR-187, which has been shown to target B7-H3 in renal cell carcinoma (Zhao et al, 2013), very little is known regarding B7-H3 and its regulation by miRNAs. This is the first study where the effect of an entire library of miRNAs on B7-H3 protein expression has been studied. We identified several miRNAs, including miR-29c, which directly targeted B7-H3 by binding to the $3^{\prime}$-UTR sequence, and demonstrated that miR-29c was significantly associated with increased survival in two cohorts of breast cancer patients.

In the initial screen, we identified nearly 50 miRNAs potentially downregulating B7-H3. The large number of hits reflects the wellknown fact that there are multiple miRNAs targeting an individual gene. In addition, many miRNAs may target other mRNAs, which then indirectly may downregulate the $\mathrm{B} 7-\mathrm{H} 3$ protein expression.

In attempts to validate the 20 most efficient miRNAs downregulating $\mathrm{B} 7-\mathrm{H} 3$ protein in the screens, we found that all the selected miRNAs led to reduced $\mathrm{B} 7-\mathrm{H} 3$ protein expression in JIMT-1 cells. For the KPL-4 cells, the effects at the B7-H3 protein level were more variable and less pronounced for some of the 

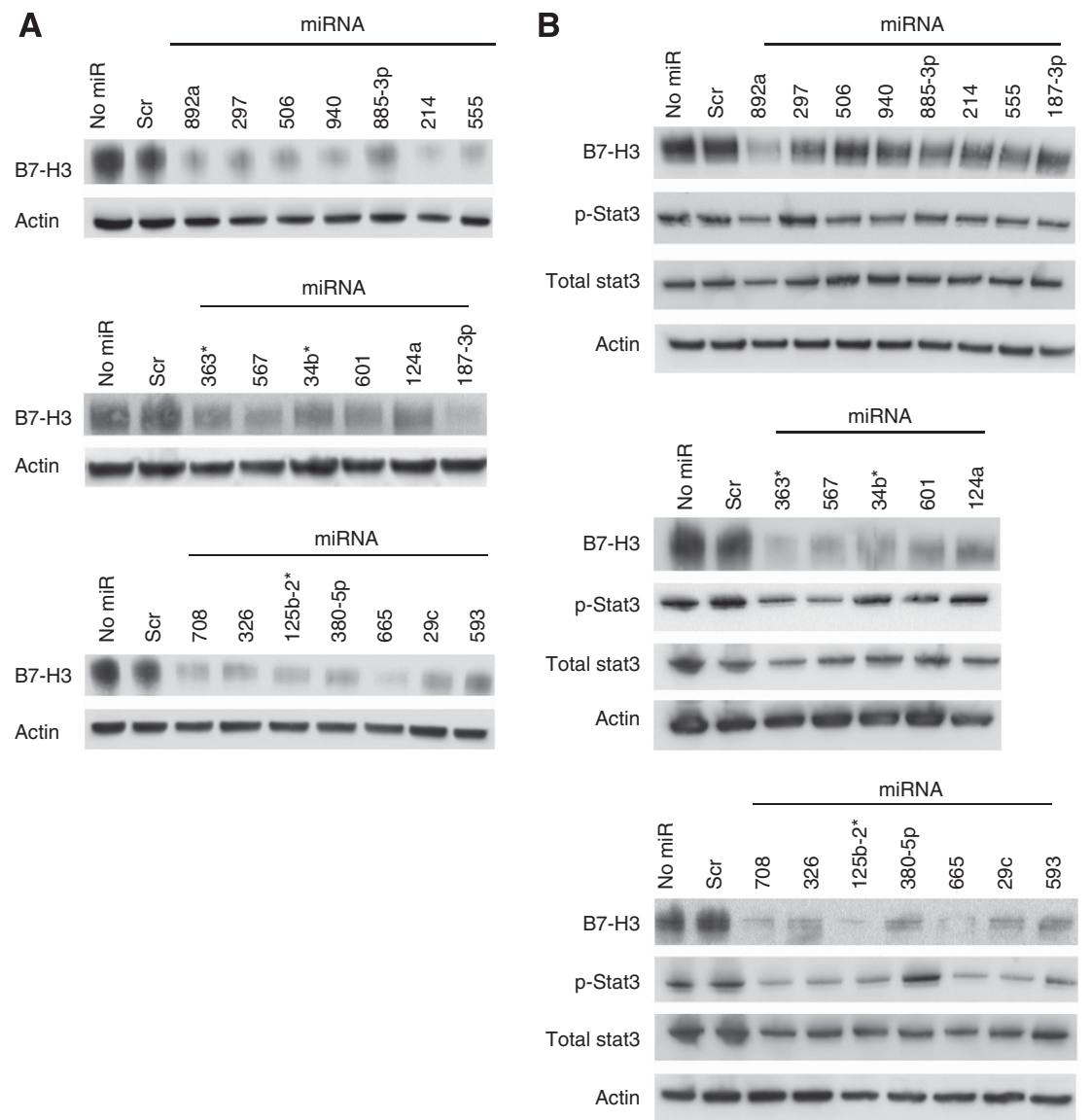

Figure 2. Validation of miRNAs regulating B7-H3. JIMT-1 (A) and KPL-4 (B) were transfected with miRVANA constructs (20 nM) and incubated for $72 \mathrm{~h}$. Thereafter, the cells were lysed and the lysates were analysed by western immunoblotting for the expression of B7-H3 (A and $\mathbf{B})$ and $\mathrm{p}$-Stat3 (B). The expression of $\beta$-actin was used as a loading control. Scr, scrambled miRNA. The experiments were repeated three (JIMT-1) or two (KPL-4) times, and representative western blots are shown.

\begin{tabular}{|c|c|c|}
\hline miRNA & Position in the B7-H3 $3^{\prime}$-UTR & Prediction algorithm \\
\hline hsa-miR-214 & $188-194$ and $257-263$ & miRanda/TargetScan \\
\hline hsa-miR-363* & $374-380$ & TargetScan \\
\hline hsa-miR-326 & $1117-1123$ & miRanda/TargetScan \\
\hline hsa-miR-940 & $253-259,398-404$ and $1035-1041$ & miRanda/TargetScan \\
\hline hsa-miR-892a & $272-278$ and 295-302 & TargetScan \\
\hline hsa-miR-297 & $278-284$ & miRanda/TargetScan \\
\hline hsa-miR-567 & $278-284$ & TargetScan \\
\hline hsa-miR-555 & $1113-1120$ & miRanda/TargetScan \\
\hline hsa-miR-29c & $1339-1346$ & miRanda/TargetScan \\
\hline hsa-miR-665 & 544-551, 1079-1085 and 1390-1396 & TargetScan \\
\hline hsa-miR-34b* & $468-474$ & TargetScan \\
\hline hsa-miR-187-3p & $416-423$ & miRanda/TargetScan \\
\hline hsa-miR-708 & $35-42$ and $1388-1394$ & miRanda/TargetScan \\
\hline
\end{tabular}

miRNAs, for example, miR-506 and miR-187-3p. MiR-506 was not predicted to bind directly to B7-H3 $3^{\prime}$-UTR, suggesting that it targets $\mathrm{B} 7-\mathrm{H} 3$ through an indirect mechanism in the JIMT-1 cell line, which may not be functioning in KPL-4 cells. Notably, several of the miRNAs that reduced B7-H3 protein levels in KPL-4 cells caused reduced phosphorylation of Stat3. Previously, it was shown that B7-H3 knockdown leads to decreased activation of Stat3 (Liu et al, 2011). MiR-892a and miR-363* clearly reduced the levels of both total Stat3 and p-Stat3, suggesting that these miRNAs might target Stat3 directly, although Stat3 was not predicted to be a direct target for these two miRNAs by two widely used target prediction algorithms, miRanda and TargetScan.

Using luciferase assays, we showed that 13 miRNAs: miR-214, miR-363*, miR-326, miR-940, miR-29c, miR-665, miR-34b ${ }^{*}$, miR-708, miR-601, miR-124a, miR-380-5p, miR-885-3p, and miR-593 directly target $\mathrm{B} 7-\mathrm{H} 3$ by associating with the B7-H3 $3^{\prime}$-UTR region, suggesting that these miRNAs have a direct role in modulating the $\mathrm{B} 7-\mathrm{H} 3$ protein expression. The fact that miR-124a, miR-380-5p, miR-885-3p and miR-593 were not predicted to bind B7-H3 3'-UTR by neither TargetScan nor miRanda, demonstrates the limitation of prediction programs and the importance of experimental validation. Interestingly, we also found that several miRNAs reduced the protein expression without binding to $\mathrm{B} 7-\mathrm{H} 3$ $3^{\prime}$-UTR. This may indicate that B7-H3 can be indirectly regulated by other target genes, or that these miRNAs do not target B7-H3 in the $3^{\prime}$-UTR but rather in other regions. It has been reported that several miRNAs exert their control on mRNAs through target sites that reside beyond the 3 '-UTR region (Lytle et al, 2007; Orom et al, 2008; Tay et al, 2008; Leivonen et al, 2011).

In both the DBCGbc cohort and the validating MicMa cohort, breast cancer-related survival was significantly increased in patients with high expression of miR-29c. In the DBCGbc cohort, higher miR-29c expression was also associated with lower risk of 


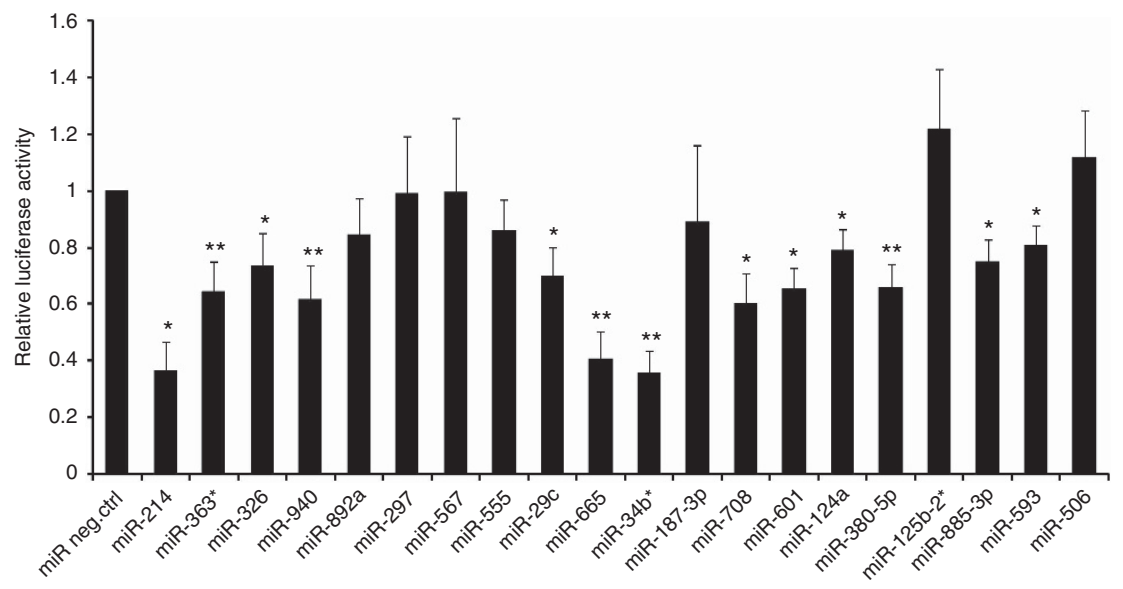

Figure 3. B7-H3 is a direct target for miR-214, miR-363*, mir-326, miR-940, miR-29c, miR-665, miR-34b*, miR-708, miR-601, miR-124a, miR-380-5p, miR-885-3p and miR-593. Activity of the luciferase reporter containing the B7-H3 3'-UTR. Reporter (100 ng) was co-transfected with miRVANA constructs $(50 \mathrm{~nm})$ for the indicated miRNAs, and the luciferase activity was measured after $24 \mathrm{~h}$ incubation. The experiments were repeated at least three times with triplicates. ${ }^{\star} P<0.05,{ }^{\star \star} P<0.01$.

A

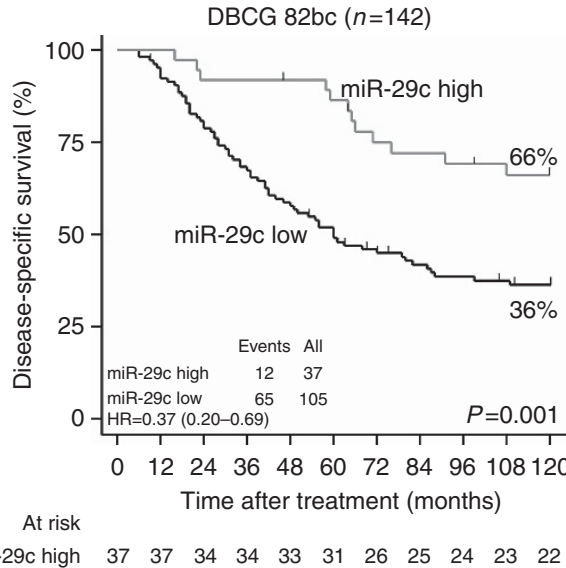

$\begin{array}{llllllllllllllll}37 & 37 & 34 & 34 & 33 & 31 & 26 & 25 & 24 & 23 & 22 & 4\end{array}$

$\begin{array}{llllllllllll}\text { miR-29c low } & 105 & 99 & 84 & 71 & 61 & 53 & 45 & 39 & 36 & 34 & 31\end{array}$
B

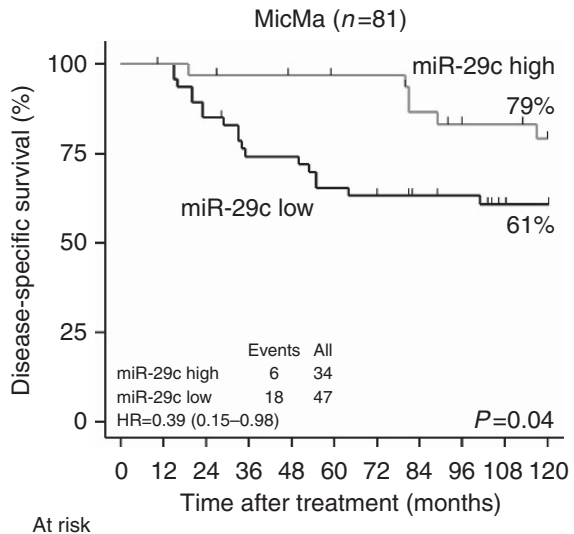

$\begin{array}{llllllllllll}\text { miR-29c high } & 34 & 33 & 32 & 31 & 30 & 29 & 29 & 25 & 23 & 22 & 20\end{array}$ miR-29c low $4 \begin{array}{lllllllllll}47 & 47 & 40 & 34 & 34 & 30 & 29 & 26 & 25 & 21 & 19\end{array}$

Figure 4. Kaplan-Meier survival curves presenting disease-specific survival (A, DBCGbc cohort; B, MicMa cohort) based on high or low expression of miR-29c.

developing distant metastasis. Several reports have demonstrated miR-29c to be important in the progression and prognosis for various cancers (Xiong et al, 2010; Zhao et al, 2010; Nguyen et al, 2011; Presneau et al, 2013), and that miR-29c enhances the sensitivity of nasopharyngeal carcinoma to cisplatin-based chemotherapy and radiotherapy (Zhang et al, 2013). To our knowledge, the present study is the first demonstrating a clinical impact of miR-29c in patients with breast cancer. It is tempting to speculate that this may at least partly be owing to reduced $\mathrm{B} 7-\mathrm{H} 3$ protein expression in these patients' tumours via a direct regulation of B7-H3 by miR-29c, and we have previously shown that B7-H3 is involved in sensitivity to chemotherapy and metastasis formation (Liu et al, 2011; Tekle et al, 2012). Interestingly, in the MicMa cohort, we found significant inverse correlation between the levels of B7-H3 transcript and miR-29c, indicating that miR-29c might affect $\mathrm{B} 7-\mathrm{H} 3$ expression also in vivo.

Although the other miRNAs that we investigated had no significant impact on breast cancer-related survival, circulating miR-125b-2* was reported to be a marker predicting chemoresistance in patients with invasive ductal carcinoma (Wang et al, 2012), and miR-34b* expression correlated negatively with diseasefree survival and overall survival in triple-negative breast cancer patients (Svoboda et al, 2012). Furthermore, several of the other
miRNAs have been linked to cancer progression and prognosis in other tumour types (Kefas et al, 2009; Saini et al, 2011; Jang et al, 2012; Majid et al, 2012; Shaham et al, 2012; Svoboda et al, 2012; Xia et al, 2012).

We also found that low expression of miR-29c was associated with TP53 mutation in both cohorts of breast cancer patients. MiR-29 has previously been linked to stabilisation of the p53 protein by targeting p $85 \alpha$ (Park et al, 2009). Accordingly, tumours with low miR-29c expression frequently have lost the TP53 tumour suppressor activity, and low miR-29-expressing tumours with wild-type TP53 gene might, nevertheless, have reduced TP53 activity owing to destabilisation of the protein. We also found that low miR-29c was associated with overexpression of HER2 and loss of ER expression in both cohorts. Neither HER2 expression nor loss of ER expression has previously been linked to miR-29c. However, as both are features of aggressive breast cancer, their association with low miR-29c corresponds well with our disease-specific survival data (high miR-29c expression predicts prolonged survival). In addition, the expression of miR-29c was significantly lower in higher grade tumours compared with lower grade tumours. miR-29c also had the lowest expression in the basal subtype, which is the most aggressive form of breast cancer. 
The molecular basis for the role of $\mathrm{B} 7-\mathrm{H} 3$ in tumour biology needs to be further elucidated, the importance of which is illustrated by the many reports showing that its expression is linked to a worsened prognosis in several cancer forms. Most studies have focused on $\mathrm{B} 7-\mathrm{H} 3$ and its role as an immunoregulatory molecule. We, however, have reported that $\mathrm{B} 7-\mathrm{H} 3$ has important roles in chemoresistance and metastasis independently of the immune system. On the basis of emerging evidence for miRNAs as regulators of cancer metastasis, it is interesting that we have identified several miRNAs targeting B7-H3 and also demonstrated significant clinical impact of one of these, miR-29c.

\section{ACKNOWLEDGEMENTS}

This work was supported by the KG Jebsen Center for Breast Cancer Research, South-Eastern Norway Regional Health Authority, the Norwegian and Danish Cancer Societies and by the University of Oslo. We thank Ellen Hellesylt for performing the B7-H3 immunohistochemistry staining.

\section{CONFLICT OF INTEREST}

The authors declare no conflict of interest.

\section{REFERENCES}

Ambros V (2001) MicroRNAs: tiny regulators with great potential. Cell 107: 823-826.

Arigami T, Narita N, Mizuno R, Nguyen L, Ye X, Chung A, Giuliano AE, Hoon DS (2010) B7-h3 ligand expression by primary breast cancer and associated with regional nodal metastasis. Ann Surg 252: 1044-1051.

Aure MR, Leivonen S-K, Fleischer T, Overgaard J, Alsner A, Louhimo R, Perälä M, Touleimat N, Tost J, Børresen-Dale A-L, Hautaniemi S, Lingjærde OC, Kleivi Sahlberg K, Kristensen VN (2013) Integrated analysis of miRNA copy number, methylation and expression data reveals novel miRNAs implicated in breast cancer. Genome Biol 14(11): R126.

Betel D, Wilson M, Gabow A, Marks DS, Sander C (2008) The microRNA.org resource: targets and expression. Nucleic Acids Res 36: D149-D153.

Collins M, Ling V, Carreno BM (2005) The B7 family of immune-regulatory ligands. Genome Biol 6: 223.

Crispen PL, Sheinin Y, Roth TJ, Lohse CM, Kuntz SM, Frigola X, Thompson RH, Boorjian SA, Dong H, Leibovich BC, Blute ML, Kwon ED (2008) Tumor cell and tumor vasculature expression of $\mathrm{B} 7-\mathrm{H} 3$ predict survival in clear cell renal cell carcinoma. Clin Cancer Res 14: 5150-5157.

Enerly E, Steinfeld I, Kleivi K, Leivonen SK, Aure MR, Russnes HG, Ronneberg JA, Johnsen H, Navon R, Rodland E, Makela R, Naume B, Perala M, Kallioniemi O, Kristensen VN, Yakhini Z, Borresen-Dale AL (2011) miRNA-mRNA integrated analysis reveals roles for miRNAs in primary breast tumors. PLoS One 6: e16915.

Ferlay J, Shin HR, Bray F, Forman D, Mathers C, Parkin DM (2010) Estimates of worldwide burden of cancer in 2008: GLOBOCAN 2008. Int J Cancer 127: 2893-2917.

Garzon R, Heaphy CE, Havelange V, Fabbri M, Volinia S, Tsao T, Zanesi N, Kornblau SM, Marcucci G, Calin GA, Andreeff M, Croce CM (2009) MicroRNA 29b functions in acute myeloid leukemia. Blood 114: 5331-5341.

Grimson A, Farh KK, Johnston WK, Garrett-Engele P, Lim LP, Bartel DP (2007) MicroRNA targeting specificity in mammals: determinants beyond seed pairing. Mol Cell 27: 91-105.

Harquail J, Benzina S, Robichaud GA (2012) MicroRNAs and breast cancer malignancy: an overview of miRNA-regulated cancer processes leading to metastasis. Cancer Biomark 11: 269-280.

Ingebrigtsen VA, Boye K, Tekle C, Nesland JM, Flatmark K, Fodstad O (2012) B7-H3 expression in colorectal cancer: nuclear localization strongly predicts poor outcome in colon cancer. Int J Cancer 131: 2528-2536.

Jang JS, Jeon HS, Sun Z, Aubry MC, Tang H, Park CH, Rakhshan F, Schultz DA, Kolbert CP, Lupu R, Park JY, Harris CC, Yang P, Jen J (2012)
Increased miR-708 expression in NSCLC and its association with poor survival in lung adenocarcinoma from never smokers. Clin Cancer Res 18: 3658-3667.

Katayama A, Takahara M, Kishibe K, Nagato T, Kunibe I, Katada A, Hayashi T, Harabuchi Y (2011) Expression of B7-H3 in hypopharyngeal squamous cell carcinoma as a predictive indicator for tumor metastasis and prognosis. Int J Oncol 38: 1219-1226.

Kefas B, Comeau L, Floyd DH, Seleverstov O, Godlewski J, Schmittgen T, Jiang J, diPierro CG, Li Y, Chiocca EA, Lee J, Fine H, Abounader R, Lawler S, Purow B (2009) The neuronal microRNA miR-326 acts in a feedback loop with notch and has therapeutic potential against brain tumors. J Neurosci 29: 15161-15168.

Kyndi M, Sorensen FB, Knudsen H, Overgaard M, Nielsen HM, Andersen J, Overgaard J (2008) Tissue microarrays compared with whole sections and biochemical analyses. A subgroup analysis of DBCG 82 b\&c. Acta Oncol 47: 591-599.

Lai EC (2002) Micro RNAs are complementary to $3^{\prime}$ UTR sequence motifs that mediate negative post-transcriptional regulation. Nat Genet 30: 363-364.

Leivonen SK, Makela R, Ostling P, Kohonen P, Haapa-Paananen S, Kleivi K, Enerly E, Aakula A, Hellstrom K, Sahlberg N, Kristensen VN, Borresen-Dale AL, Saviranta P, Perala M, Kallioniemi O (2009) Protein lysate microarray analysis to identify microRNAs regulating estrogen receptor signaling in breast cancer cell lines. Oncogene 28: 3926-3936.

Leivonen SK, Rokka A, Ostling P, Kohonen P, Corthals GL, Kallioniemi O, Perala M (2011) Identification of miR-193b targets in breast cancer cells and systems biological analysis of their functional impact. Mol Cell Proteomics 10: M110 005322.

Leivonen SK, Sahlberg KK, Makela R, Due EU, Kallioniemi O, Borresen-Dale AL, Perala M (2014) High-throughput screens identify microRNAs essential for HER2 positive breast cancer cell growth. Mol Oncol 8: 93-104.

Liu C, Liu J, Wang J, Liu Y, Zhang F, Lin W, Gao A, Sun M, Wang Y, Sun Y (2013) B7-H3 expression in ductal and lobular breast cancer and its association with IL-10. Mol Med Rep 7: 134-138.

Liu H, Tekle C, Chen YW, Kristian A, Zhao Y, Zhou M, Liu Z, Ding Y, Wang B, Maelandsmo GM, Nesland JM, Fodstad O, Tan M (2011) B7-H3 silencing increases paclitaxel sensitivity by abrogating Jak2/Stat3 phosphorylation. Mol Cancer Ther 10: 960-971.

Lytle JR, Yario TA, Steitz JA (2007) Target mRNAs are repressed as efficiently by microRNA-binding sites in the $5^{\prime}$ UTR as in the $3^{\prime}$ UTR. Proc Natl Acad Sci USA 104: 9667-9672.

Majid S, Dar AA, Saini S, Shahryari V, Arora S, Zaman MS, Chang I, Yamamura S, Tanaka Y, Chiyomaru T, Deng G, Dahiya R (2012) MicroRNA-34b inhibits prostate cancer through demethylation, active chromatin modifications and AKT pathways. Clin Cancer Res 19: 73-84.

Myhre S, Mohammed H, Tramm T, Alsner J, Finak G, Park M, Overgaard J, Borresen-Dale AL, Frigessi A, Sorlie T (2010) In silico ascription of gene expression differences to tumor and stromal cells in a model to study impact on breast cancer outcome. PLoS One 5: e14002.

Nguyen T, Kuo C, Nicholl MB, Sim MS, Turner RR, Morton DL, Hoon DS (2011) Downregulation of microRNA-29c is associated with hypermethylation of tumor-related genes and disease outcome in cutaneous melanoma. Epigenetics 6: 388-394.

Orom UA, Nielsen FC, Lund AH (2008) MicroRNA-10a binds the 5'UTR of ribosomal protein mRNAs and enhances their translation. Mol Cell 30: 460-471.

Park SY, Lee JH, Ha M, Nam JW, Kim VN (2009) miR-29 miRNAs activate p53 by targeting p85 alpha and CDC42. Nat Struct Mol Biol 16: 23-29.

Presneau N, Eskandarpour M, Shemais T, Henderson S, Halai D, Tirabosco R, Flanagan AM (2013) MicroRNA profiling of peripheral nerve sheath tumours identifies miR-29c as a tumour suppressor gene involved in tumour progression. Br J Cancer 108: 964-972.

Roth TJ, Sheinin Y, Lohse CM, Kuntz SM, Frigola X, Inman BA, Krambeck AE, McKenney ME, Karnes RJ, Blute ML, Cheville JC, Sebo TJ, Kwon ED (2007) B7-H3 ligand expression by prostate cancer: a novel marker of prognosis and potential target for therapy. Cancer Res 67: 7893-7900.

Saini S, Yamamura S, Majid S, Shahryari V, Hirata H, Tanaka Y, Dahiya R (2011) MicroRNA-708 induces apoptosis and suppresses tumorigenicity in renal cancer cells. Cancer Res 71: 6208-6219.

Selbach M, Schwanhausser B, Thierfelder N, Fang Z, Khanin R, Rajewsky N (2008) Widespread changes in protein synthesis induced by microRNAs. Nature 455: 58-63. 
Shaham L, Binder V, Gefen N, Borkhardt A, Izraeli S (2012) MiR-125 in normal and malignant hematopoiesis. Leukemia 26: 2011-2018.

Shenouda SK, Alahari SK (2009) MicroRNA function in cancer: oncogene or a tumor suppressor? Cancer Metastasis Rev 28: 369-378.

Sun TW, Gao Q, Qiu SJ, Zhou J, Wang XY, Yi Y, Shi JY, Xu YF, Shi YH, Song K, Xiao YS, Fan J (2012) B7-H3 is expressed in human hepatocellular carcinoma and is associated with tumor aggressiveness and postoperative recurrence. Cancer Immunol Immunother 61: 2171-2182.

Sun Y, Wang Y, Zhao J, Gu M, Giscombe R, Lefvert AK, Wang X (2006) B7-H3 and B7-H4 expression in non-small-cell lung cancer. Lung Cancer 53: 143-151.

Svoboda M, Sana J, Redova M, Navratil J, Palacova M, Fabian P, Slaby O, Vyzula R (2012) MiR-34b is associated with clinical outcome in triple-negative breast cancer patients. Diagn Pathol 7: 31.

Tay Y, Zhang J, Thomson AM, Lim B, Rigoutsos I (2008) MicroRNAs to Nanog, Oct 4 and Sox 2 coding regions modulate embryonic stem cell differentiation. Nature 455: 1124-1128.

Tekle C, Nygren MK, Chen YW, Dybsjord I, Nesland JM, Maelandsmo GM, Fodstad O (2012) B7-H3 contributes to the metastatic capacity of melanoma cells by modulation of known metastasis-associated genes. Int J Cancer 130: 2282-2290.

Valencia-Sanchez MA, Liu J, Hannon GJ, Parker R (2006) Control of translation and mRNA degradation by miRNAs and siRNAs. Genes Dev 20: $515-524$.

Wang H, Tan G, Dong L, Cheng L, Li K, Wang Z, Luo H (2012) Circulating $\mathrm{MiR}-125 \mathrm{~b}$ as a marker predicting chemoresistance in breast cancer. PLoS One 7: e34210.

Wang J, Chong KK, Nakamura Y, Nguyen L, Huang SK, Kuo C, Zhang W, Yu H, Morton DL, Hoon DS (2013) B7-H3 associated with tumor progression and epigenetic regulatory activity in cutaneous melanoma. J Invest Dermatol 133: 2050-2058.

Wiedswang G, Borgen E, Karesen R, Kvalheim G, Nesland JM, Qvist H, Schlichting E, Sauer T, Janbu J, Harbitz T, Naume B (2003) Detection of isolated tumor cells in bone marrow is an independent prognostic factor in breast cancer. J Clin Oncol 21: 3469-3478.

Xia H, Ooi LL, Hui KM (2012) MiR-214 targets beta-catenin pathway to suppress invasion, stem-like traits and recurrence of human hepatocellular carcinoma. PLoS One 7: e44206.
Xiong Y, Fang JH, Yun JP, Yang J, Zhang Y, Jia WH, Zhuang SM (2010) Effects of microRNA-29 on apoptosis, tumorigenicity, and prognosis of hepatocellular carcinoma. Hepatology 51: 836-845.

Xu H, Cheung IY, Guo HF, Cheung NK (2009) MicroRNA miR-29 modulates expression of immunoinhibitory molecule B7-H3: potential implications for immune based therapy of human solid tumors. Cancer Res 69: 6275-6281.

Yamato I, Sho M, Nomi T, Akahori T, Shimada K, Hotta K, Kanehiro H, Konishi N, Yagita H, Nakajima Y (2009) Clinical importance of B7-H3 expression in human pancreatic cancer. Br J Cancer 101: 1709-1716.

Yuan H, Wei X, Zhang G, Li C, Zhang X, Hou J (2011) B7-H3 over expression in prostate cancer promotes tumor cell progression. J Urol 186: 1093-1099.

Zang X, Sullivan PS, Soslow RA, Waitz R, Reuter VE, Wilton A, Thaler HT, Arul M, Slovin SF, Wei J, Spriggs DR, Dupont J, Allison JP (2010) Tumor associated endothelial expression of $\mathrm{B} 7-\mathrm{H} 3$ predicts survival in ovarian carcinomas. Mod Pathol 23: 1104-1112.

Zhang JX, Qian D, Wang FW, Liao DZ, Wei JH, Tong ZT, Fu J, Huang XX, Liao YJ, Deng HX, Zeng YX, Xie D, Mai SJ (2013) MicroRNA-29c enhances the sensitivities of human nasopharyngeal carcinoma to cisplatin-based chemotherapy and radiotherapy. Cancer Lett 329: 91-98.

Zhao J, Lei T, Xu C, Li H, Ma W, Yang Y, Fan S, Liu Y (2013) MicroRNA-187, down-regulated in clear cell renal cell carcinoma and associated with lower survival, inhibits cell growth and migration though targeting B7-H3. Biochem Biophys Res Commun 438: 439-444.

Zhao JJ, Lin J, Lwin T, Yang H, Guo J, Kong W, Dessureault S, Moscinski LC, Rezania D, Dalton WS, Sotomayor E, Tao J, Cheng JQ (2010) microRNA expression profile and identification of miR-29 as a prognostic marker and pathogenetic factor by targeting CDK6 in mantle cell lymphoma. Blood 115: $2630-2639$.

This work is published under the standard license to publish agreement. After 12 months the work will become freely available and the license terms will switch to a Creative Commons AttributionNonCommercial-Share Alike 3.0 Unported License.

Supplementary Information accompanies this paper on British Journal of Cancer website (http://www.nature.com/bjc) 